UTHEP-480

UTCCP-P-144

\title{
Non-perturbative renormalization of vector and axial vector currents in quenched QCD for a renormalization group improved gauge action *
}

\author{
CP-PACS Collaboration: K. Ide, ${ }^{\mathrm{a}}$ S. Aoki, ${ }^{\mathrm{a}}$ M. Fukugita, ${ }^{\mathrm{b}}$ N. Ishizuka, ${ }^{\mathrm{a}, \mathrm{c}}$ Y. Iwasaki ${ }^{\mathrm{a}, \mathrm{c}}$ K. Kanaya, \\ T. Kaneko, ${ }^{d}$ Y. Kuramashi, ${ }^{d}$ V. Lesk, M. Okawa ${ }^{\mathrm{e}}$ Y. Taniguchi, ${ }^{\mathrm{a}}$ A. Ukawa ${ }^{\mathrm{a}, \mathrm{c}}$ and T. Yoshié ${ }^{\mathrm{a}, \mathrm{c}}$

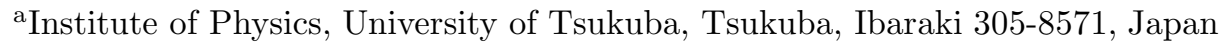 \\ ${ }^{\mathrm{b}}$ Institute for Cosmic Ray Research, University of Tokyo, Kashiwa, Chiba 277-8582, Japan \\ ${ }^{\mathrm{c}}$ Center for Computational Physics, University of Tsukuba, Tsukuba, Ibaraki 305-8577, Japan \\ ${ }^{\mathrm{d}}$ High Energy Accelerator Research Organization (KEK), Tsukuba, Ibaraki 305-0801, Japan \\ ${ }^{\text {e}}$ Department of Physics, Hiroshima University, Higashi-Hiroshima, Hiroshima 739-8526, Japan
}

Renormalization constants of vector $\left(Z_{V}\right)$ and axial-vector $\left(Z_{A}\right)$ currents are determined non-perturbatively in quenched QCD for an RG-improved gauge action and a tadpole-improved clover quark action using the Schrödinger functional method. Meson decay constants $f_{\rho}$ and $f_{\pi}$ show much better scaling when $Z_{V}$ and $Z_{A}$ estimated for infinite physical volume are used instead of $Z$-factors from tadpole-improved one-loop perturbation theory.

\section{Introduction}

In a recent comprehensive study by the $\mathrm{CP}$ PACS Collaboration of $N_{f}=2$ full QCD [1], meson decay constants were found to exhibit a very large scaling violation over the range of lattice spacing $a^{-1} \approx 1-2 \mathrm{GeV}$. This was disappointing since an RG-improved gluon action and SheikoleslamiWohlert quark action with tadpole-improved $c_{\mathrm{sw}}$ were used. In this calculation, however, one-loop perturbative $Z$-factors, albeit tadpole-improved, were used for currents. A natural question was whether scaling becomes improved if nonperturbative $Z$-factors are employed instead.

At Lattice2001, we reported an initial study of this problem using the Schrödinger functional (SF) method 2] within quenched QCD. We found the problem of anomalously large values appearing in the ensemble of hadron correlators toward strong coupling where CP-PACS data of decay constants had been taken. In this report, we have analyzed this problem in some detail. Here we present our final results on the $Z$-factors including these analysis.

\footnotetext{
*Talk presented by K. Ide.
}

\section{Method}

We follow the method developed by the ALPHA collaboration [3], and work with a lattice geometry of $L^{3} \times T$ with $T=2 L$ for $Z_{V}$ with a vector operator at $t=L$, and for $Z_{A}$ with two axial vector operators at $t=3 T / 8$ and $t=5 T / 8$.

Tree-level values are used for the coefficients of boundary counter terms of the action. For improving the axial current, we adopt the one-loop perturbative value for the coefficient $c_{A}$.

Values of $Z_{V}$ and $Z_{A}$ are determined for $\beta=$ $2.2-8.0$ which approximately covers the range of the CP-PACS quenched calculation [1, $\beta=2.187$ -2.575 . We have analyzed 200-20000 configurations depending on $\beta$ value and lattice size.

\section{Exceptional Configurations}

It is straight-forward to calculate $Z$-factors for $\beta \geq 2.6$. For lower $\beta$ values on large lattices such as $8^{3} \times 16$, however, anomalously large values appear in the ensemble of hadron correlators. This makes it difficult to determine quark mass precisely, and since this means uncertainties in $\kappa_{c}$, also that of $Z$-factors. 


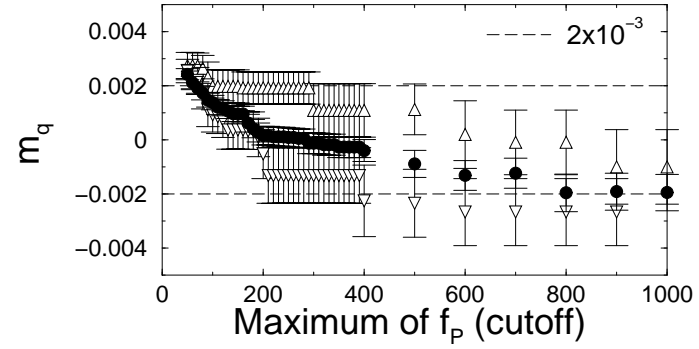

Figure 1. Cutoff dependence of $m_{q}$ at $\beta=2.4$ on an $8^{3} \times 16$ lattice for three $\kappa$ 's around $\kappa_{c}$.

We suspect that these "exceptional" configurations are an artifact of quenched approximation; having very small or negative eigenvalues of the Wilson-Dirac operator, they would be suppressed in full QCD. Since one cannot distinguish "exceptional" configurations from "normal" ones on some rigorous basis, we restrict the configurations used for averaging to those having the value of a relevant hadron correlator below some cutoff. We then examine if uncertainties under variation of the cutoff are contained within some acceptable magnitude.

In Fig. 1we illustrate this test for $m_{q}$ for which a cutoff is set for $f_{P}$ (see Ref. 3] for definition). We estimate $\kappa_{c}$ from $m_{q}$ with the cutoff value of 300 , because $m_{q}$ is rather stable there. The uncertainty in $m_{q}$ at the $\kappa_{c}$ is $\approx \pm 2 \times 10^{-3}$, once the cutoff of $f_{P}$ is taken in the range $200-1000$.

In Fig. 2 we show how much the $Z$-factors depend on $m_{q} . Z_{V}$ is insensitive to $m_{q}$, and $Z_{A}$ is consistent within $10 \%$ or so, albeit apparently exhibiting a more pronounced dependence.

We analyze the uncertainties in the statistical averaging of $Z$-factors themselves by applying a cutoff in $f_{1}$, as carried out in Ref. 2]. The conclusion is similar; $Z_{V}$ is very stable against variation of the cutoff, and $Z_{A}$ shows a more conspicuous variation of $5 \%$ or so.

Uncertainties of $Z_{A}$ on an $8^{3}$ lattice of order $15 \%$ in total lead to uncertainty of $Z_{A}$ normalized at infinite volume of order $30 \%$. The uncertainty, however, has little effect in a Padé fit of $Z_{A}$ and hence final results; $Z_{A}$ varies less than $3 \%$ at the largest coupling $\beta=2.187$, even if we artificially shift $Z_{A}$ at $\beta=2.4$ by $30 \%$.

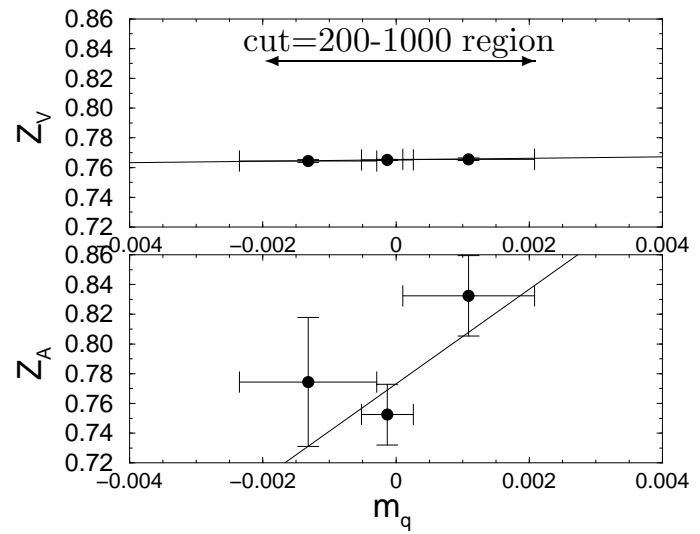

Figure 2. $m_{q}$ dependence of $Z_{V}$ and $Z_{A}$ at $\beta=$ 2.4 on an $8^{3} \times 16$ lattice.
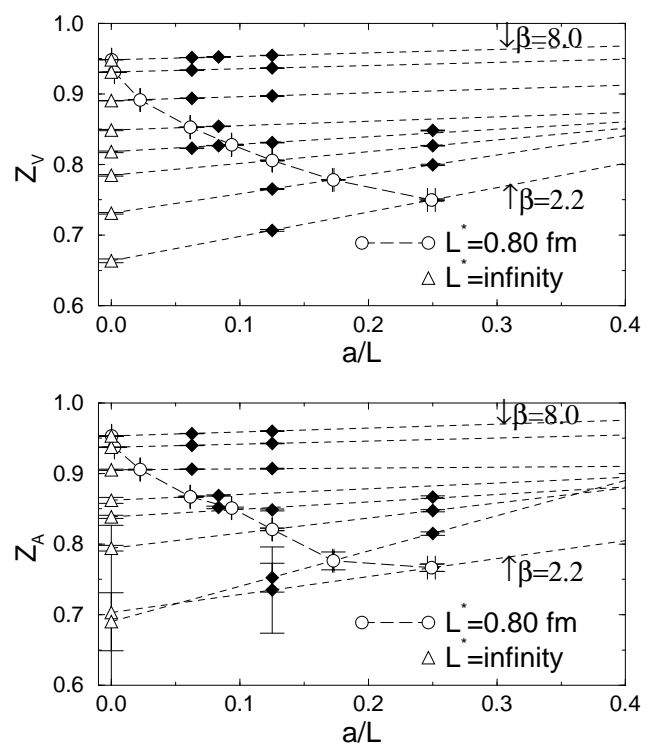

Figure 3. Size dependence of $Z_{V}$ and $Z_{A}$.

\section{Results for $Z$-factors}

We determine the $Z$-factors for infinite volume $\left(L^{*}=\infty\right)$ and also for a fixed finite physical volume $\left(L^{*}=0.8 \mathrm{fm}\right.$ corresponding to $8^{3}$ lattice at $\beta=2.6$ ) for comparison. The lattice scale is set through the string tension

As shown in Fig. 3] size dependence of $Z$ factors becomes sizable toward strong couplings. Since our quark action employs a tadpoleimproved value of $c_{\mathrm{sw}}$, we expect $O(a)$ errors in the $Z$-factors. Therefore we extrapolate or inter- 

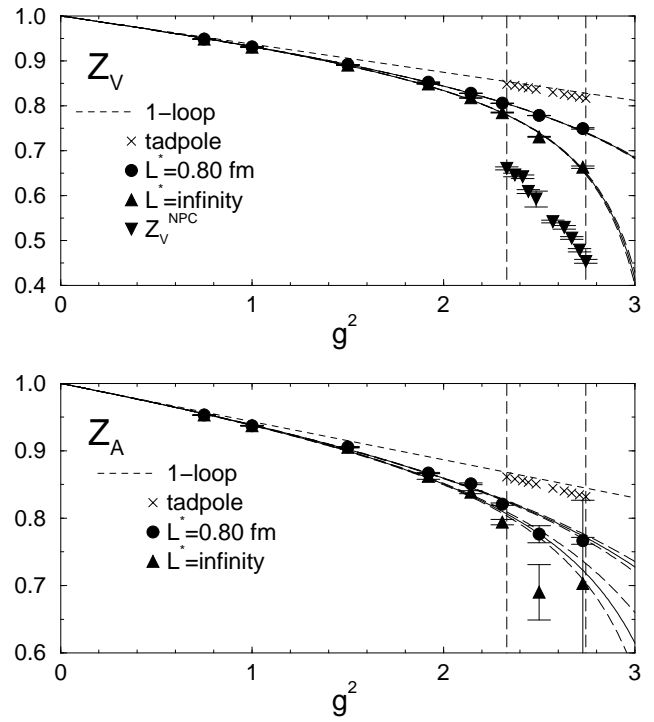

Figure 4. Results for $Z_{V}$ and $Z_{A}$ normalized at $L^{*}=0.8 \mathrm{fm}$ and $L^{*}=\infty$.

polate results linearly in $a / L$ to obtain estimates at $L^{*}=0.8 \mathrm{fm}$ and at $L^{*}=\infty$.

In Fig. 4 we show results of $Z$-factors as a function of bare coupling $g^{2}$, together with Padé fits (solid curves in the figure) to them. Nonperturbative estimates give values smaller than the one-loop perturbative ones (dashed lines) by about $20 \%(15 \%)$ for $Z_{V}\left(Z_{A}\right)$ at the largest coupling of the CP-PACS simulation, $\beta=2.187$.

$Z_{V}^{N P C}$ determined from the ratio of the conserved vector current to the local one differs significantly from $Z_{V}$ from the SF method, because the local current is not $O(a)$-improved.

\section{Scaling Property of Decay Constants}

We compare in Fig. $5 f_{\pi}$ and $f_{\rho}$ determined with non-perturbative $Z$-factors normalized at $L^{*}=\infty$ (filled circles) with those using perturbative $Z$-factors (open up triangles). For comparison, open squares are the results from the standard plaquette and Wilson action 4 .

We observe a very encouraging result that with the non-perturbative $Z$-factors scaling violations are sizably reduced. Furthermore the continuum extrapolation yields values consistent with those from the standard action.

In the same figure, we overlay $f_{\pi}$ and $f_{\rho}$ deter-
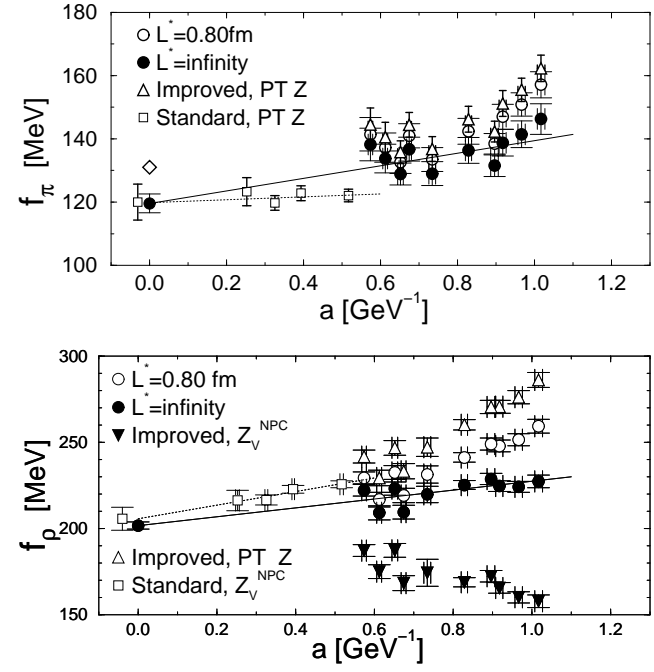

Figure 5. $f_{\pi}$ and $f_{\rho}$ vs. $a$ for our improved action with non-perturbative and perturbative (PT) $Z$ factors together with results for the standard action 4 .

mined with $Z$-factors normalized at finite $L^{*}=$ $0.8 \mathrm{fm}$ (open circles). Scaling is best improved when $Z$-factors are normalized at $L^{*}=\infty$. This property is likely related to the fact that $O(a / L)$ errors in $Z$-factors are removed in the limiting procedure $L^{*} \rightarrow \infty$.

We also find that $f_{\rho}$ determined from the conserved vector current (filled down triangle in Fig. (5) exhibits a large scaling violation.

This work is supported in part by Grants-inAid of the Ministry of Education (Nos. 12304011, $12640253, \quad 13135204,13640260, \quad 14046202$, 14740173, 15204015, 15540251, 15540279 ).

\section{REFERENCES}

1. CP-PACS collaboration: A. Ali Khan et al., Phys. Rev. D65 (2002) 054505.

2. CP-PACS collaboration: S. Aoki et al., Nucl. Phys. B (Proc. Suppl.) 106 (2002) 844

3. M. Lüscher et al., Nucl. Phys. B491 (1997) 344.

4. CP-PACS collaboration: S. Aoki et al., Phys. Rev. Lett. 84 (2000) 238; Phys. Rev. D67 (2003) 034503.

5. M. Lüscher et al., Nucl. Phys. B384 (1992) 168. 